\title{
Studies of challenge in lower hybrid current drive capability at high density regime in experimental advanced superconducting tokamak
}

\author{
B. J. Ding ${ }^{1} \dagger$, M. H. Li ${ }^{1}$, Y. C. Li ${ }^{1}$, M. Wang ${ }^{1}$, F. K. Liu ${ }^{1}$, J. F. Shan ${ }^{1}$, \\ J. G. Li $^{1}$, B. N. Wan ${ }^{1}$ and for EAST team and collaborators \\ ${ }^{1}$ Institute of Plasma Physics, Chinese Academy of Sciences, Hefei 230031, PR China \\ (Received 5 July 2016; revised 31 December 2016; accepted 4 January 2017)
}

Aiming at a fusion reactor, two issues must be solved for the lower hybrid current drive (LHCD), namely good lower hybrid wave (LHW)-plasma coupling and effective current drive at high density. For this goal, efforts have been made to improve LHW-plasma coupling and current drive capability at high density in experimental advanced superconducting tokamak (EAST). LHW-plasma coupling is improved by means of local gas puffing and gas puffing from the electron side is taken as a routine way for EAST to operate with LHCD. Studies of high density experiments suggest that low recycling and high lower hybrid (LH) frequency are preferred for LHCD experiments at high density, consistent with previous results in other machines. With the combination of $2.45 \mathrm{GHz}$ and $4.6 \mathrm{GHz} \mathrm{LH}$ waves, a repeatable high confinement mode plasma with maximum density up to $4.5 \times 10^{19} \mathrm{~m}^{-3}$ was obtained by LHCD in EAST. In addition, in the first stage of LHCD cyclic operation, an alternative candidate for more economical fusion reactors has been demonstrated in EAST and further work will be continued.

Key words: magnetized plasmas, plasma heating, plasma production

\section{Introduction}

Lower hybrid current drive (LHCD) (Fisch 1978; Bernabei et al. 1982; Fisch 1987) plays a key role in controlling the current profile in tokamak experiments aimed at improving confinement and stability of fusion plasmas. The first high confinement (H-mode) plasma by LHCD was obtained in limiter configuration in JT-60 (Tusji et al. 1990). In joint European torus (JET), a long lasting internal transport barrier (ITB) was sustained during H-mode operation using LHCD (Crisanti et al. 2002; Cesario et al. 2004). Recently, the LHCD H-mode has been extensively obtained in EAST (Xu et al. 2011; Ding et al. 2013a,b; Liu et al. 2015; Wan et al. 2015). However, concerning a fusion reactor, at least two issues must be solved, namely a good lower hybrid wave (LHW)-plasma coupling and effective current drive capability at high density, since high density is inevitable in fusion reactors. As mentioned in the following sections, related works have been studied and continued in many fusion

\footnotetext{
$†$ Email address for correspondence: bjding@ipp.ac.cn
} 


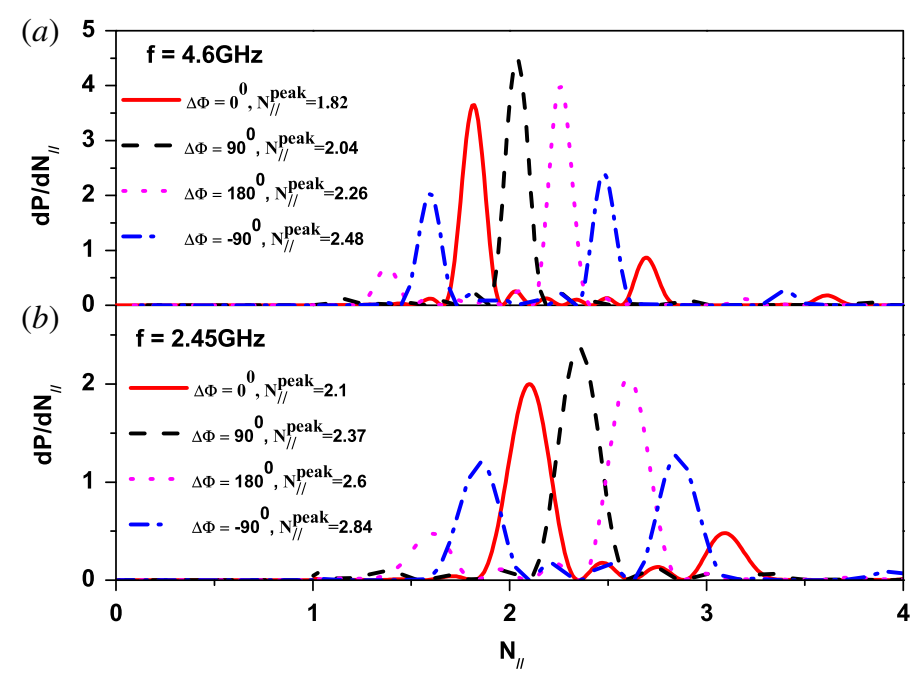

FIGURE 1. Typical spectra for the $4.6 \mathrm{GHz}(a)$ and $2.45 \mathrm{GHz}(b) \mathrm{LH}$ antennas calculated by the ALOHA code $\left(N_{/ /}\right.$is the refractive index in parallel direction, $N_{/ /}^{\text {peak }}$ is the corresponding peak value, $\Delta \Phi$ refers to the phase difference between the adjacent main waveguides. The directivity defined by the ratio of power with positive $n_{/ /}$to total power is $74 \%-75 \%$ and $76 \%-77 \%$ for $2.45 \mathrm{GHz}$ and $4.6 \mathrm{GHz}$, respectively.).

devices, including Japan tokamak-60 upgrade (JT-60U), axially symmetric divertor experiment (ASDEX), JET, Tore-supra, Hefei tokamak-7 (HT-7), Alcator C-Mod and frascati tokamak upgrade (FTU).

In order to obtain the scientific goal of EAST (a long pulse and high performance), two LHCD systems have been developed and exploited on EAST, operating, respectively, at $2.45 \mathrm{GHz}$ (4 MW) (Ding et al. 2011, 2015) and $4.6 \mathrm{GHz}$ (6 MW) (Ding et al. 2013a,b). Typical spectra calculated by an advanced lower hybrid antenna (ALOHA) (Hillairet et al. 2010) are shown in figure 1, in which $N_{/ /}$is the refractive index in the parallel direction, $N_{/ /}^{\text {peak }}$ is the corresponding peak value and $\Delta \Phi$ refers to the phase difference between the adjacent main waveguides. For clarity, the spectrum with $0 \leqslant N_{/ /} \leqslant 4$ is displayed. Aiming at exploring the role of LHCD in a fusion reactor, related studies in EAST including LHW-plasma coupling and current drive capability toward to high density have been performed and are summarized as follows.

\section{Issue of LHW-plasma coupling}

Good LHW-plasma coupling is the first necessary condition for LHCD experiments, especially for sustaining a long pulse H-mode. In the H-mode plasma, the edge density will decrease rapidly enough that the density at the grill mouth does not satisfy the wave-plasma coupling condition (Brambilla 1976; Petržilka et al. 1991), resulting in a large reflection. Consequently, the LHW power cannot be effectively coupled to the plasma if the plasma density at the grill is below the cutoff density.

To improve LHW-plasma coupling, local gas puffing near the antenna has been developed, which has been studied in JT-60U, ASDEX, JET, Tore-supra, HT-7 (Leuterer et al. 1991; Ikeda et al. 1994; Pericoli-Ridolfini et al. 2004; Ekedahl et al. 2005, 2009, 2012; Ding et al. 2010). Also, in EAST, with the $2.45 \mathrm{GHz}$ LHCD system, local gas puffing from the electron drift side (GIM_e) and ion drift side 


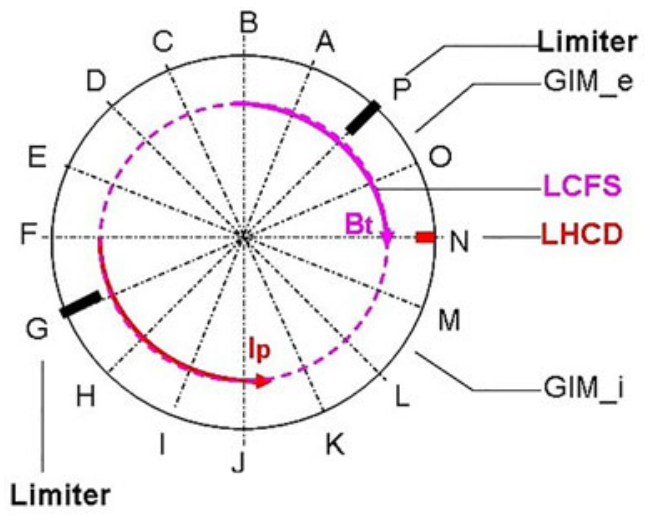

FIGURE 2. Top view of local gas puffing for electron drift side and ion drift side.

(a)

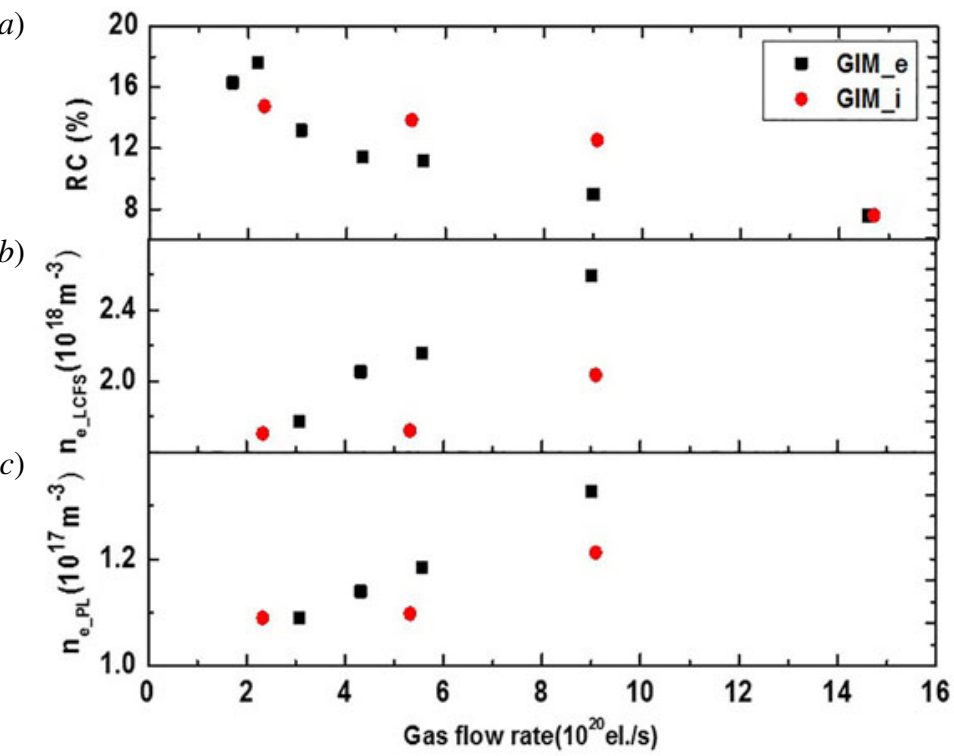

FIGURE 3. Coupling comparison between gas puffing from electron side (GIM_e) and ion side (GIM_i) (a) reflection coefficient, $(b)$ density at the last closed flux surface (LCFS) and $(c)$ density at the poloidal limiter $(f=0.45 \mathrm{GHz})$.

(GIM_i), as shown in figure 2, was studied (Ding et al. 2013a,b). The experimental results are shown in figure 3, indicating the effect of gas puffing on coupling improvement from the electron drift side is better than that from the ion drift side. It is seen that, for the same gas flow, the reflection coefficient (RC) in the case of GIM_i gas puffing is a little higher, implying that the density at the grill mouth in this case is less favourable to the coupling. This is also demonstrated by the measured edge density. The difference in the edge density between electron- and ion-side cases suggests that local ionization of puffed gas plays a dominant role in affecting the grill density due to different movement direction of ionized electrons and that part of 


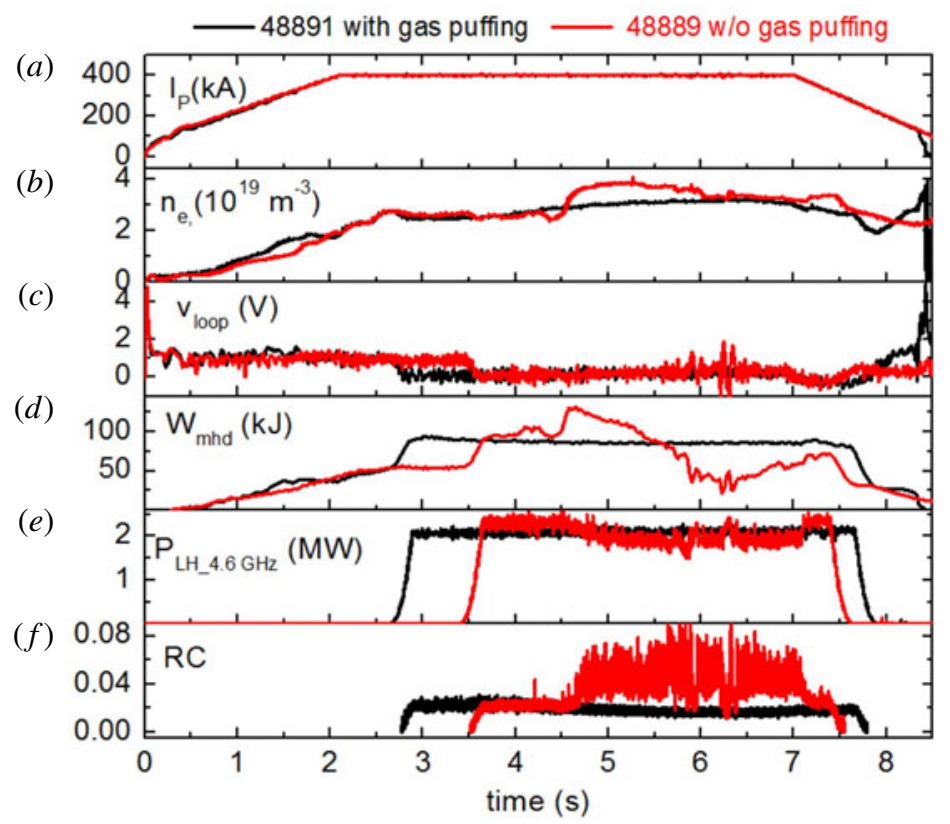

Figure 4. Coupling improved by means of local gas puffing ( $f=4.6 \mathrm{GHz})$. (a) Plasma current $\left(I_{p}\right)$, $(b)$ central-line-averaged density $\left(n_{e}\right)$, $(c)$ loop voltage $\left(V_{\text {loop }}\right),(d)$ stored energy $\left(W_{m h d}\right)$ calculated with magnetic measurement, $(e)$ internal inductance (li) and (f) reflection coefficient $(\mathrm{RC})$.

gas has been locally ionized near the gas pipe before diffusing into the grill region (Ding et al. 2013a,b).

Referenced from the above study, only local gas puffing structures from the electron drift side were installed on EAST, both for $2.45 \mathrm{GHz}$ and $4.6 \mathrm{GHz}$, so as to save the space in the device. Similar to the $2.45 \mathrm{GHz}$ LHCD, as shown in figure 4 , it is seen that the coupling with the $4.6 \mathrm{GHz} \mathrm{LHW}$ is improved by means of local gas puffing. Also, the coupling characteristics between low confinement (L-mode) and Hmode are also compared in figure 5. It is seen that, although the $\mathrm{RC}$ in the H-mode is higher than that in the L-mode, its level remains acceptable and does not much affect the coupling. Therefore, in routine experiments in EAST, local gas puffing from the electron drift side is utilized for good LHW-plasma coupling.

\section{LHCD capability at high density}

High density is a challenge with LHCD experiments. Reactor relevant high plasma density regimes have faced the LHCD tool with the challenge of effectively penetrating the main plasma without excessive power dissipation of the coupled radio frequency (RF) power at the edge. LHCD experiments on many tokamaks (Pericoli-Ridolfini, Giannone \& Bartiromo 1994; Cesario et al. 2010; Wallace et al. 2010; Cesario et al. 2011) have shown a decrease in non-thermal tails and a drop in bremsstrahlung emission much steeper than $1 / n_{e}$ at high densities, which is stronger than expected based on current models. Similar results (Ding et al. 2013a,b, 2015) have also been reported in EAST. Some possible mechanisms, including collisional absorption (CA) (Bonoli \& Englade 1986), parametric instabilities (PI) (Liu \& 


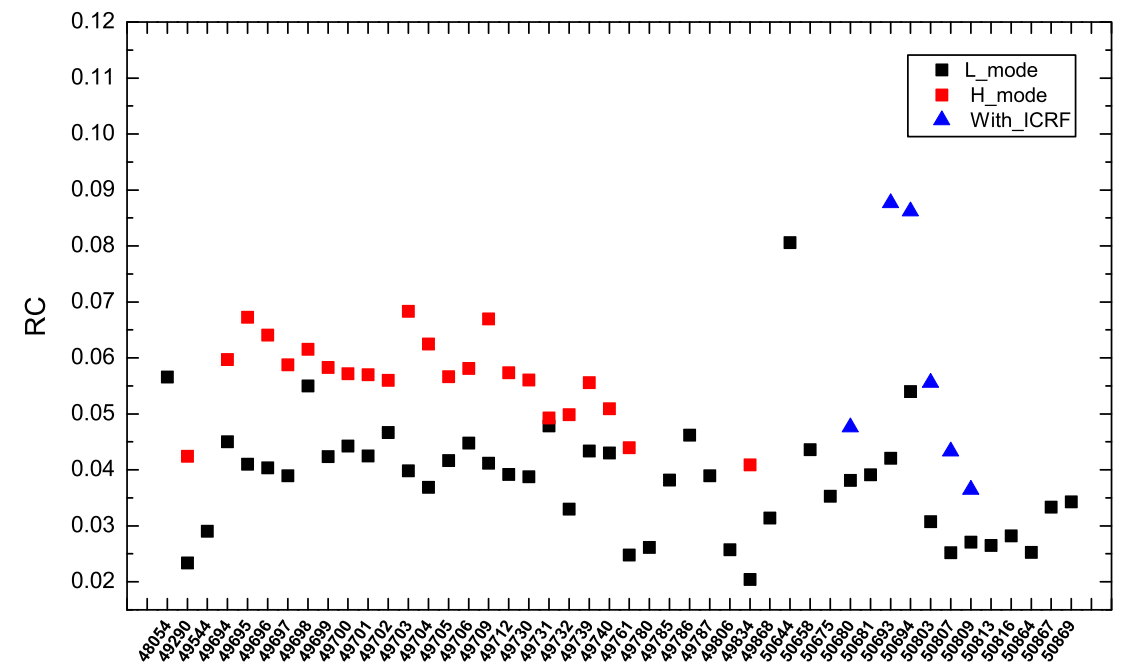

FIGURE 5. Coupling characteristics comparison between L- and H-mode $(f=4.6 \mathrm{GHz})$.

Tripathi 1986; Cesario et al. 2004) and scattering from density fluctuations (SDF) (Bonoli \& Ott 1982; Peysson et al. 2011), individually or simultaneously, have been identified to preclude the penetration of LHWs, dissipating the power in the plasma periphery and degrading LH efficiency. In order to explore the experimental condition for high current drive (CD) efficiency at high density in EAST, experiments with a double null configuration with the $2.45 \mathrm{GHz}$ LHW $\left(N_{/ /}^{\text {peak }}=2.1\right)$ were studied (Ding et al. 2013a,b) by increasing density in one discharge with poor and strong lithiation, following the method assessed on FTU of the reduced PI effect under the higher temperature of the plasma edge (Cesario et al. 2004, 2010). In the experiments, the local gas puffing from GIM_e is utilized. The capability of the current drive is estimated by the count of hard X-ray rate $(60-200 \mathrm{keV})$ normalized by the injected LHW power, which is proportional to the current driven efficiency. Seen from the results shown in figure $6(a)$, in strongly lithiated discharge, there is no sudden decrease in driven current, where the hard X-ray radiation (HXR) counts deviate from the curve of $1 / n_{e}$, until a density of up to $3.0 \times 10^{19} \mathrm{~m}^{-3}$, which is much larger than the value of $2.0 \times 10^{19} \mathrm{~m}^{-3}$ in the case of poor lithiation. This suggests that the strong wall lithiation promotes the occurrence of the LHCD effect at high density, in agreement with previous results (Cesario et al. 2010).

The trends of LHCD with plasma density and PI signatures, documented by the frequency power spectrum of the signal collected by a RF probe located outside the machine, have been analysed. The spectrum consists a broadening at approximately the LH wave operating frequency, and a sideband shift of an amount in the range of the ion cyclotron frequency (IC sideband). The relationship between IC sideband and plasma density is shown in figure $6(b)$. It shows that, compared to the strong lithiation case, the IC sideband frequency in the case of poor lithiation decreases faster with density to a low value of $\sim 14.5 \mathrm{MHz}$, which corresponds to the local IC frequency in the edge region where PI occurs. The trend of decreasing IC sideband frequency with density is consistent with the sharp decay of HXR counts that indicates the current drive capability, supporting the conclusion that the PI mechanism plays a key role in affecting LHCD. The measured RF spectrum indicating PI behaviour is nearly in 


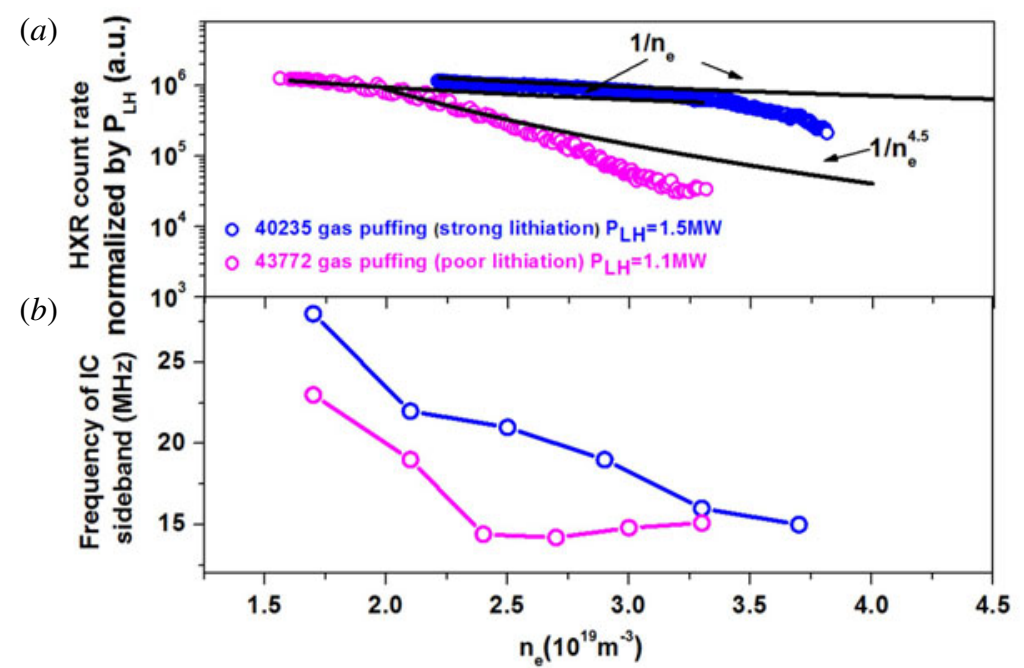

FIgURE 6. (a) Relationship between HXR counts and density with strong and poor lithiation, $(b)$ frequency of IC sidebands with strong and poor lithiation.

agreement the PI modelling based on the PI code (Takase \& Porkolab 1983; Cesario et al. 2011; Baek et al. 2014), demonstrating the influence of PI on LHCD efficiency in EAST (Ding et al. 2015). Studies indicate that low recycling is preferred for high density LHCD operation and, more generally, for improving confinement and stability (Cesario et al. 2004, 2010, 2013, 2014).

\section{Effect of LH frequency on CD characteristic}

LHW frequency is one of important parameters affecting wave propagation. In order to study the effect of frequency on LHCD characteristics, two different frequency waves $(2.45 \mathrm{GHz}$ and $4.6 \mathrm{GHz})$ with the same power $\left(P_{L H}=1.05 \mathrm{MW}\right)$ were injected in one discharge with almost constant density $\left(n_{e}=2.0 \times 10^{19} \mathrm{~m}^{-3}\right)$ and the typical waveforms are shown in figure 7 . It is seen that the residual voltages $\left(V_{\text {loop }}\right)$ are $0.27 \mathrm{~V}$ and $0.15 \mathrm{~V}$ respectively for a current drive with $2.45 \mathrm{GHz}$ and $4.6 \mathrm{GHz}$, indicating better $\mathrm{CD}$ efficiency for $4.6 \mathrm{GHz}$ wave. Better plasma heating effect for 4.6 GHz wave can be obtained from the time evolution of plasma stored energy $\left(W_{M H D} \sim 68.3 \mathrm{~kJ}\right.$ and $74.8 \mathrm{~kJ}$, respectively for $2.45 \mathrm{GHz}$ and $4.6 \mathrm{GHz}$ ). Also, the internal inductance is higher with the $4.6 \mathrm{GHz} \mathrm{LH}$ wave injection, indicating the occurrence of more effective LHCD in the plasma core with respect to the $2.45 \mathrm{GHz}$ wave (Hooke 1984; Takase et al. 1985). Since the power spectrum is an important parameter affecting wave propagation and power deposition, using a ray-tracing/Fokker-Planck code (C3PO/LUKE) (Peysson \& Decker 2008; Peysson, Decker \& Morini 2012) with the experimental spectra $\left(N_{/ /}^{\text {peak }}=2.1\right.$ and 2.04 for $2.45 \mathrm{GHz}$ and $4.6 \mathrm{GHz}$, respectively), power deposition and driven current profile are calculated without considering the spectrum broadening (see figure 8). It is seen that though the total driven current is somewhat small with the $2.45 \mathrm{GHz} \mathrm{LHW}$, it cannot account for the experimental discrepancy $(\sim 100 \mathrm{kA})$ estimated by the loop voltage. Therefore, the discrepancy in the initial spectrum could not dominate the difference in the current drive. In addition, a comparison of frequency spectra between the two 


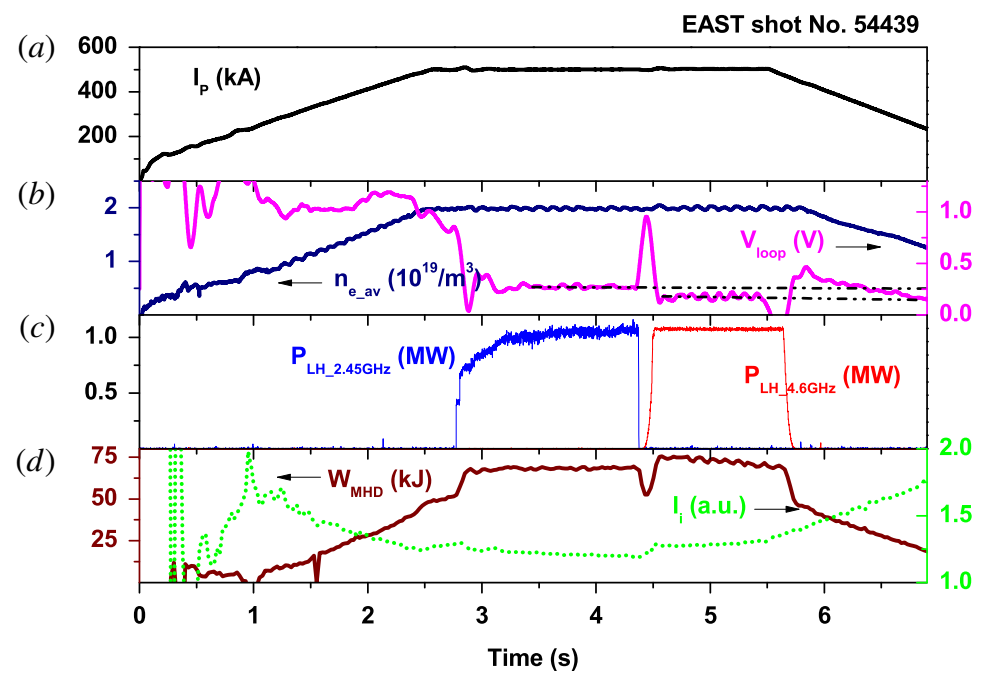

FIGURE 7. Effect of LH frequency on LHCD characteristics. (a) Plasma current $\left(I_{p}\right)$, (b) central-line-averaged density $\left(n_{e}\right)$ and loop voltage $\left(V_{\text {loop }}\right),(c)$ LH power, $(d)$ stored energy $\left(W_{m h d}\right)$ calculated with magnetic measurement and internal inductance (li).
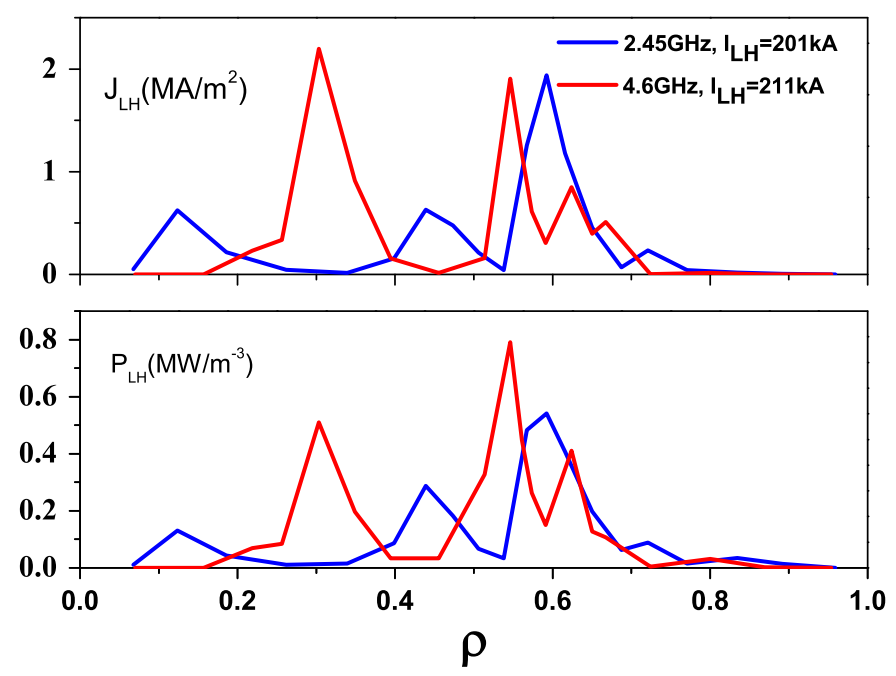

FIGURE 8. Power simulation and drive current profile with C3PO/LUKE code.

waves is illustrated in figure 9, from which it is seen that larger spectral broadening occurs for the $2.45 \mathrm{GHz}$ case, indicating a stronger PI behaviour. This could partly explain the better CD effect with the $4.6 \mathrm{GHz} \mathrm{LH}$ wave in terms of reduced parasitic PI effect, as previously reported (Cesario et al. 2004, 2010).

The above results indicate that the $4.6 \mathrm{GHz}$ frequency is more efficient for producing a stronger LHCD on EAST. Based on the studies of coupling and LHCD characteristics, a repeatable H-mode was obtained by LHCD in EAST. With the $2.45 \mathrm{GHz}$ and $4.6 \mathrm{GHz}$ LHCD systems, a high density H-mode is obtained and the 


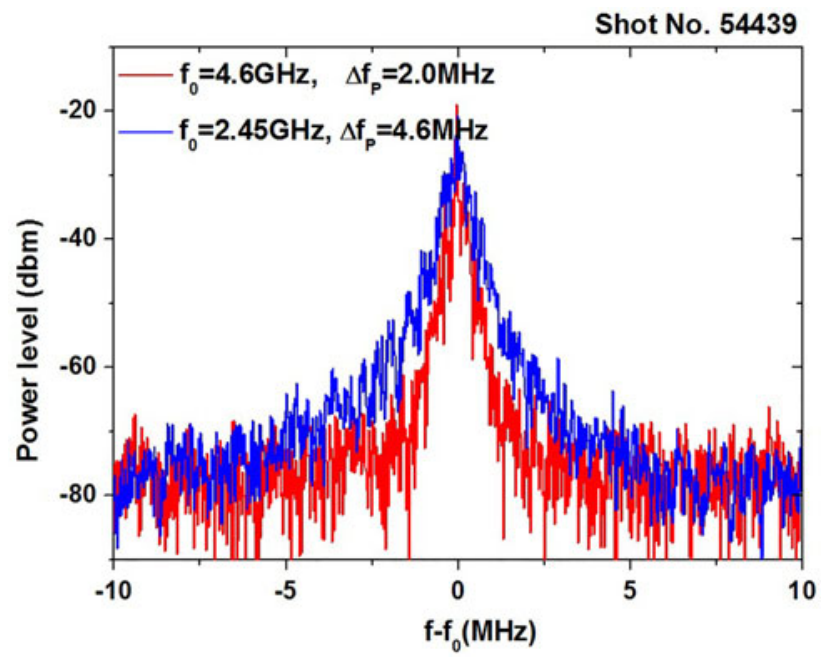

FIGURE 9. Spectrum broadening measured by a RF loop antenna.
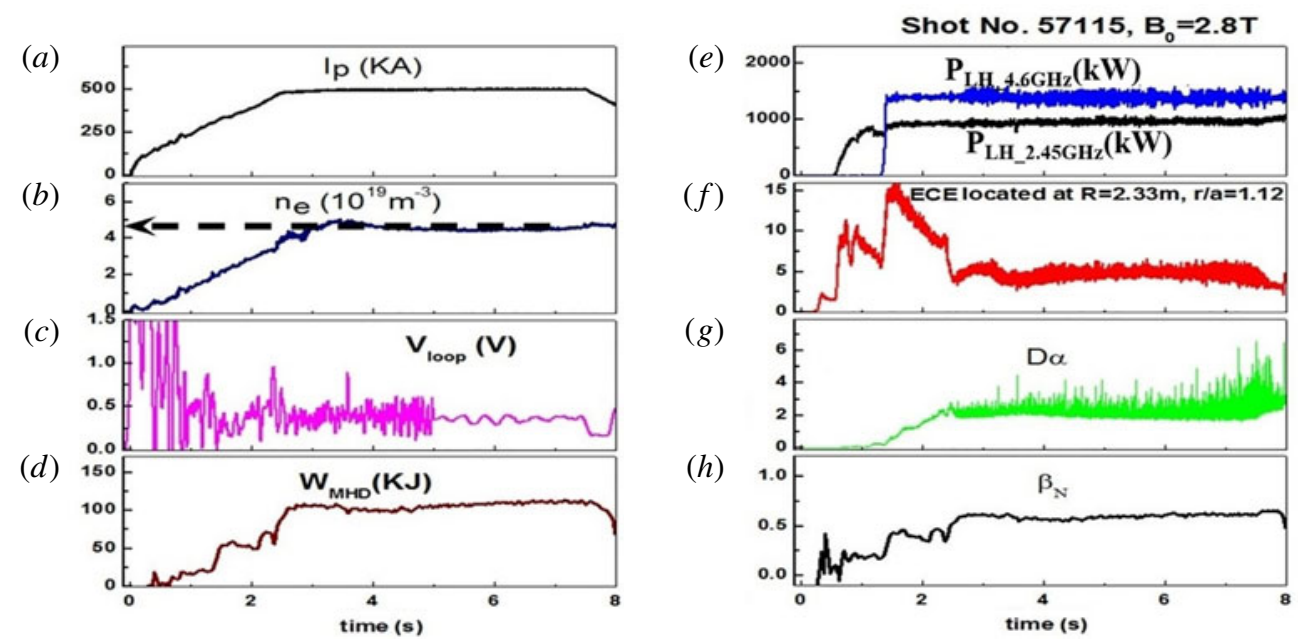

FIgURE 10. Typical LHCD H-mode plasma at high density. (a) Plasma current $\left(I_{p}\right)$, (b) central-line-averaged density $\left(n_{e}\right),(c)$ loop voltage $\left(V_{\text {loop }}\right),(d)$ stored energy $\left(W_{\text {mhd }}\right)$ calculated with magnetic measurement, $(e)$ LH power $(f)$ ECE supra-electron emission measured by ECE in edge region, $(g)$ neutral hydrogen radiation $(\mathrm{Da}),(h)$ normalized beta $\left(\beta_{N}\right)$.

typical waveforms are shown in figure 10. The electron cyclotron emission (ECE) signal drops quickly at the L-H transition, implying a decrease of LH driven current, possibly due to the concomitant density increase. Seen from the loop voltage and ECE signal, it is inferred that part of current is driven by LHW even if at the density of $4.5 \times 10^{19} \mathrm{~m}^{-3}$. Such a density is higher than that of $1.5-2.5 \times 10^{19} \mathrm{~m}^{-3}$ obtained in the $2.45 \mathrm{GHz}$ case (Ding et al. 2013a,b).

Concerning the application of the $\mathrm{LH}$ wave to a reactor, whether in the higher frequency range or the lower, in which many alpha particles could be in the plasma 
centre, there is a concern that energetic alpha particles in the plasma centre could damp the lower hybrid wave. This was first pointed out by Wong \& Ono (1984), supported by calculations by Fisch \& Rax (1992a), and led to suggestions (Barbato \& Santini 1991; Pavlo, Krlin \& Tluchor 1991) that, to propagate LH waves close to the plasma centre while avoiding absorption of the LH wave by alpha particles, it would be necessary to use LH waves in a rather high frequency range, even higher than the $4.6 \mathrm{GHz}$ used here, in EAST tokamak. The waveguides for launching such high frequency waves are technologically more challenging than those used at lower frequency.

However, the calculations of the energetic alpha particle damping (Wong \& Ono 1984; Barbato \& Santini 1991; Pavlo et al. 1991; Fisch \& Rax 1992a) do not take into account that the wave-particle interaction occurs in a radial gradient of the alpha particles. Because the alpha particles in the centre are energetic, but cool at the periphery, the alpha particles can amplify the LH waves through the alpha channelling effect (Fisch \& Rax 1992b). Hence, it might not be necessary to resort to very high frequencies to allow the LH waves to reach near the plasma centre in a reactor, for two reasons: first, from the consideration of interaction with alpha particles, instead of the wave damping on the alpha particles, the wave might grow due to the interaction with alpha particles. That would also enable the wave to counteract the electron Landau damping that tends to extinguish the LH wave near the very hot tokamak centre, allowing further penetration than in the absence of the channelling effect. Second, the alpha channelling effect allows the tokamak to operate in the hot ion mode, where the electrons can be made much colder than the ions (Fisch \& Herrmann 1994). Among the advantages of operating at lower electron temperature is that the Landau damping of the LHW by electrons is lessened, allowing further penetration to the tokamak centre. If the wave damping concerns were alleviated for these two reasons, then the main concerns on the frequency would be only the linear wave accessibility and the avoidance of the PI.

Although the alpha channelling effect has generally been pursued using waves in the ion cyclotron range of frequencies (Fisch 1995; Herrmann \& Fisch 1997), recently it was shown that there are ways of launching the LH wave that can exploit the alpha channelling effect (Ochs, Bertelli \& Fisch 2015a,b). The preferred launching techniques avoid launching the LH wave from the low field side of the tokamak. One might then also imagine using the LHW to accomplish synergistic alpha channelling effects when more than one wave is used (Fisch \& Herrmann 1995). The alpha channelling effect can also be accomplished synergistically with the cyclic operation of LHCD, or transformer recharging, considered in the next section (Fisch 2010).

\section{Cyclic operation of lower hybrid current drive}

Due to the density limitation of LHCD, at which the CD efficiency decreases sharply, LHCD cyclic operation (Fisch 1987) could be one alternative candidate for more economical fusion reactors. The schematic of the cyclic operation regime is shown in figure 11, in which there are two stages: the current generation stage, namely the current ramp-up by LH, and the current relaxation stage.

The first stage is characterized by plasma with relatively low density and high effective ion charge state $Z_{\text {eff }}$, so that the plasma current can be driven effectively by $\mathrm{LH}$, and simultaneously the current can be ramped up quickly, due to (with larger $Z_{\text {eff }}$ or smaller electron temperature) the relatively short time constant $L / R$, where $L$ and $R$ are the plasma inductance and the resistivity, respectively. With the current 


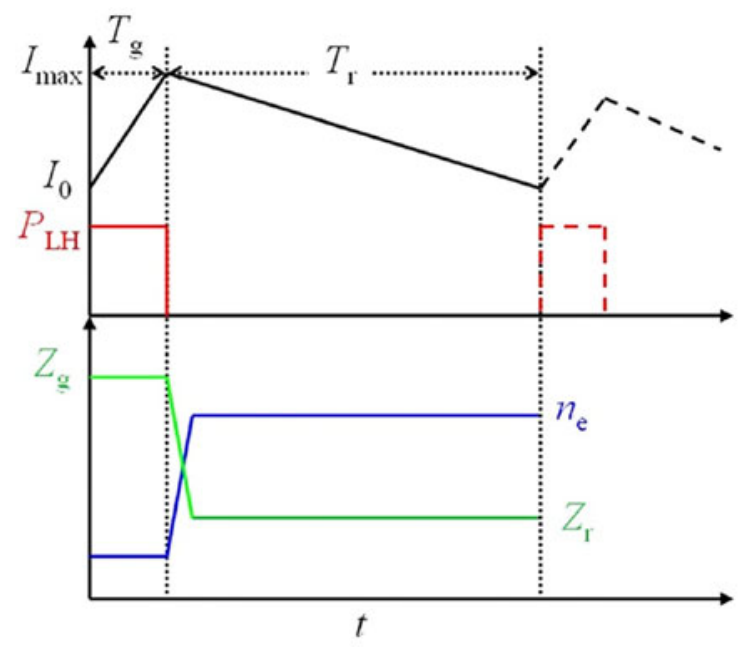

FIGURE 11. Schematic of LHCD cyclic operation. ( $I_{0}$ is the plasma current before LH application and $I_{\max }$ is the maximum plasma current before switching off LH power. $T_{g}$ and $T_{r}$ are time durations, and $Z_{g}$ and $Z_{r}$ are the effective ion charge states for the two stages, respectively.).

ramped up, the LH power would be converted into poloidal field energy stored in plasma column and then released in the current relaxation stage. During the current relaxation stage, the plasma would be optimized by higher density for greater fusion power and lower effective ion charge state for longer relaxation time. Therefore, there are at least 3 key points for the LHCD cyclic operation, (i) fast over-drive at low density and slow decay at high density, (ii) zero ohmic field completely and (iii) density and impurity control.

In EAST, the experiments (Ding et al. 2012) for the first stage have been carried out and the preliminary results are shown in figure 12, for the first time demonstrating current ramp-up without ohmic heating and transformer recharging in EAST. The plasma current was ramped up with a time-averaged rate of $18 \mathrm{kA} / \mathrm{s}$ with $\mathrm{LH}$ power. The average conversion efficiency $P_{e l} / P_{L H}$ was approximately $3 \%$, where $P_{e l}$ is the increase in poloidal field energy due to the $\mathrm{LH}$ contribution and $P_{L H}$ is the $\mathrm{LH}$ power.

\section{Conclusion and discussion}

Aiming at a fusion reactor, efforts have been made to improve LHW-plasma coupling and current drive capability at high density in EAST as follows. (i) LHW-plasma coupling is improved by means of local gas puffing and gas puffing from the electron side is taken as a routine procedure for EAST operation with LHCD. (ii) High density experiments suggest that, due to the PI effect and collisional absorption in the edge region, high edge temperature and low edge density are preferred for the LHCD experiments at high density. (iii) High LH frequency is useful to improve $\mathrm{RF}$ power propagation into the core region and enhance $\mathrm{CD}$ efficiency at high density. A repeatable H-mode plasma is obtained by LHCD. Currently, the $\mathrm{H}$-mode with maximum density (of $4.5 \times 10^{19} \mathrm{~m}^{-3}$ ) is obtained with the combination of the two LHCD systems $(2.45 \mathrm{GHz}$ and $4.6 \mathrm{GHz}$ ). (iv) The first stage of LHCD cyclic operation has been demonstrated in EAST. 

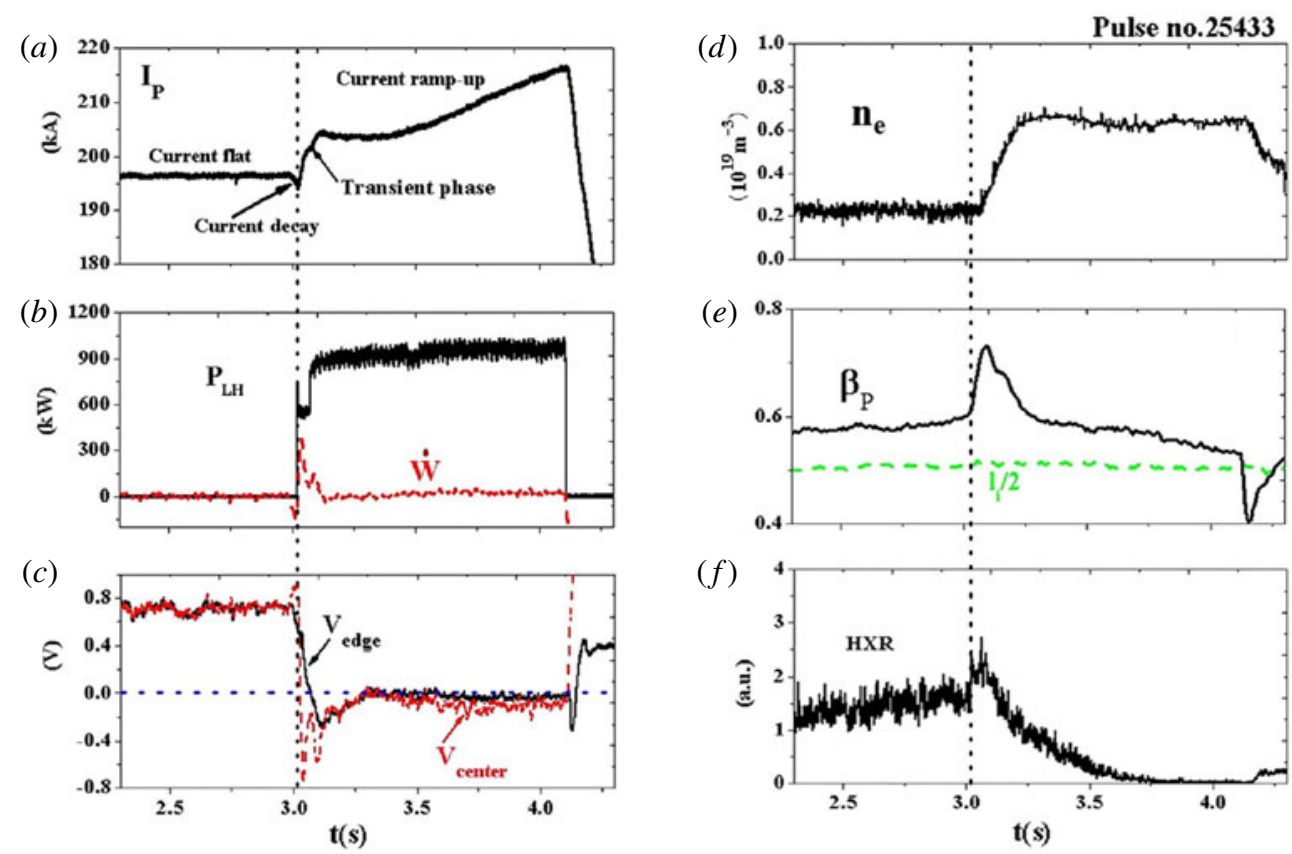

FIGURE 12. Typical time traces of current ramp-up by LH (see Ding et al. 2012). (a) Plasma current $\left(I_{\mathrm{p}}\right),(b)$ injected LH power $\left(P_{L H}\right)$ and the rate of change of poloidal field energy, $(c)$ loop voltage in edge $\left(V_{\text {edge }}\right)$ and centre $\left(V_{\text {centre }}\right),(d)$ line-averaged density $\left(n_{e}\right),(e)$ poloidal beta $\left(\beta_{p}\right)$ and half of the internal inductance $\left(l_{i} / 2\right)$ and $(f)$ hard X-ray radiation (HXR).

As mentioned above, although some progress has been made in LHW-plasma coupling and LHCD capability at high density, further work should be continued. (i) Although the density can be improved by local gas puffing, feedback control of the grill density by gas puffing is a challenge, especially for long pulse LHCD operation. (ii) The mechanisms affecting $\mathrm{CD}$ efficiency show that edge parameters play an important role. However, it is not easy to effectively control the parameters since coupling and current drive both depend on edge parameters. (iii) Although preliminary results show that high $\mathrm{LH}$ frequency is useful to improve LHCD capability at high density, the mechanism is not so clear and further studies need to be continued. (iv) Although the first stage has been demonstrated in EAST, some challenges still exist for the operation. For example, (a) it is very difficult to turn off the ohmic field completely, since it and the equilibrium field are coupled to each other, and (b) the ramp-up rate is relatively slow because the $L / R$ times are relatively large due to the large size of EAST. To fix the second issue, one possible way is to increase the drive current $\left(I_{L H}\right)$, or reduce $L / R$ by increasing the impurity. (v) In addition, high temperatures in the edge region should be very important for LHCD operation at high density. As done in the experiments, lowering recycling by means of lithiation could be one effective way. Another could be the direct heating of electrons near the edge region, as concluded in Cesario et al. (2010). Since electron cyclotron resonance heating is available in EAST, it could be an effective tool to enhance current drive capability at high density. 


\section{Acknowledgements}

This work is supported by the National Magnetic Confinement Fusion Science Program of China (grant nos 2015GB102003, 2013GB106001B, 2013GB112003), the National Natural Science Foundation of China under grant nos 11175206, 11305211, 11275233 and 11675214, Hefei Science Center CAS (2015HSC-UE005, 2016HSC-IU008) and the JSPS-NRF-NSFC A3 Foresight Program in the field of Plasma Physics (NSFC no. 11261140328). It is partly supported by the China-France, the China-Italy and the China-US Collaboration program.

\section{REFERENCES}

Baek, S. G., Parker, R. R., Shiraima, S., Wallace, G. M., Bonoli, P. T., Porkolab, M., TAkase, Y., Brunner, D., Faust, I. C., Hubbard, A. E. et al. 2014 Characterization of the onset of ion cyclotron parametric decay instability of lower hybrid waves in a diverted tokamak. Phys. Plasmas 21, 061511.

BARbato, E. \& SANTINI, F. 1991 Quasi-linear absorption of lower hybrid waves by fusion generated alpha particles. Nucl. Fusion 31, 673-685.

Bernabei, S., Daughney, C., Efthimion, P., Hooke, W., Hosea, J., Jobes, F., Martin, A., Mazzucato, E., Meservey, E., Motley, R. et al. 1982 Lower-hybrid current drive in the PLT tokamak. Phys. Rev. Lett. 49, 1255-1258.

Bonoli, P. T. \& Englade, R. C. 1986 Simulation model for lower hybrid current drive. Phys. Fluids 29, 2937-2950.

BonOLI, P. T. \& OTT, E. 1982 Toroidal and scattering effects on lower-hybrid wave propagation. Phys. Fluids 25, 359-375.

BRAMBILLA, M. 1976 Slow-wave launching at the lower hybrid frequency using a phased waveguide array. Nucl. Fusion 16, 47-54.

Cesario, R., Amicucci, L., Cardinali, A., Castaldo, C., Marinucci, M., Napoli, F., Paoletti, F., De Arcangelis, D., Ferrari, M., Galli, A. et al. 2014 Spectral broadening of parametric instability in lower hybrid current drive at a high density. Nucl. Fusion 54, 043002 .

Cesario, R., Amicucci, L., Cardinali, A., Castaldo, C., Marinucci, M., Panaccione, L., Santini, F., Tudisco, O., Apicella, M. L., Calabrò, G. et al. 2010 Current drive at plasma densities required for thermonuclear reactors. Nat. Commun. 55, 1-8.

Cesario, R., Amicucci, L., Castaldo, C., Kempenatrs, M., Jachmich, S., Mailloux, J., Tudisco, O., Galli, A., Krivska, A. \& JET-EFDA Contributors 2011 Plasma edge density and lower hybrid current drive in JET (Joint European Torus). Plasma Phys. Control. Fusion 53, 085011.

Cesario, R., Amicucci, L., Fonseca, A., Chapman, I. T., Jenko, F., Marinucci, M., SaArelma, S., Smeulders, P., Told, D., Zagorski, R. et al. 2013 Low-recycling conditions and improved core confinement in steady-state operation scenarios in JET (Joint European Torus). Plasma Phys Control. Fusion 55, 045005.

Cesario, R., Cardinali, A., Castaldo, C., Paoletti, F. \& Mazon, D. 2004 Modeling of a lower-hybrid current drive by including spectral broadening induced by parametric instability in tokamak plasmas. Phys. Rev. Lett. 92, 175002.

Crisanti, F., Litaudon, X., Mailloux, J., Mazon, D., Barbato, E., Baranov, Y., Bécoulet, A., Bécoulet, M., Challis, C. D., Conway, G. D. et al. 2002 JET quasistationary internaltransport-barrier operation with active control of the pressure profile. Phys. Rev. Lett. 88, 145004.

Ding, B. J., Kong, E. H., Li, M. H., Zhang, L., Wei, W., Wang, M., Xu, H. D., Li, Y. C., LING, B. L., ZANG, Q. et al. 2013 a Experimental investigations of LHW-plasma coupling and current drive related to achieving H-mode plasmas in EAST. Nucl. Fusion 53, 113027.

Ding, B. J., Kong, E. H., Zhang, T., Ekedahl, A., Li, M. H., Zhang, L., Wei, W., Li, Y. C., WU, J. H., XU, G. S. et al. $2013 b$ Effect of gas puffing from different side on lower hybrid 
wave-plasma coupling in experimental advanced superconductive tokamak. Phys. Plasmas 20, 102504.

Ding, B. J., LI, M. H., Fisch, N., Qin, H., Li, J. G., Wilson, J. R., Kong, E. H., Zhang, L., WEI, W., LI, Y. C. et al. 2012 Current ramp-up with lower hybrid current drive in EAST. Phys. Plasmas 19, 122507.

Ding, B. J., Li, M. H., Qin, Y. L., Li, W. K., Zhang, L. Z., Shan, J. F., LiU, F. K., Wang, M., Meng, L. G., XU, H. D. et al. 2010 Investigations of lower hybrid wave-plasma coupling by gas puffing in HT-7. Phys. Plasmas 17, 022504.

Ding, B. J., LI, Y. C., Zhang, L., Li, M. H., Wei, W., Kong, E. H., Wang, M., Xu, H. D., WANG, S. L., XU, G. S. et al. 2015 Investigations of LHW-plasma coupling and current drive at high density related to H-mode experiments in EAST. Nucl. Fusion 55, 093030.

Ding, B. J., QIn, Y. L., LI, W. K., LI, M. H., Kong, E. H., Zhang, L., Ekedahl, A., Peysson, Y., DECKER, J. \& WANG, M. 2011 Investigation of lower hybrid wave coupling and current drive experiments at different configurations in experimental advanced superconducting tokamak. Phys. Plasmas 18, 082510.

Ekedahl, A., Granucci, G., Mailloux, J., Baranov, Y., Erents, S. K., Joffrin, E., Litaudon, X., Loarte, A., Lomas, P. J., Mcdonald, D. C. et al. 2005 Long distance coupling of lower hybrid waves in JET plasmas with edge and core transport barriers. Nucl. Fusion 45, 351-359.

Ekedahl, A., Petrzilka, V., Baranov, Y., Biewer, T. M., Brix, M., Goniche, M., Jacquet, P., Kirov, K. K., Klepper, C. C., MaillouX, J. et al. 2012 Influence of gas puff location on the coupling of lower hybrid waves in JET ELMy H-mode plasmas. Plasma Phys. Control. Fusion 54, 074004.

Ekedahl, A., Rantamäki, K., Goniche, M., Mailloux, J., Petrzilka, V., Alper, B., Baranov, Y., Basiuk, V., Beaumont, P., Corrigan, G. et al. 2009 Effect of gas injection during LH wave coupling at ITER-relevant plasma-wall distances in JET. Plasma Phys. Control. Fusion 51, 044001.

FISCH, N. J. 1978 Confining a tokamak plasma with rf-driven currents. Phys. Rev. Lett. 41, 873-876.

FISCH, N. J. 1987 Theory of current drive in plasmas. Rev. Mod. Phys. 59, 175-234.

FISCH, N. J. 1995 Alpha power channeling using ion-Bernstein waves. Phys. Plasma 2, 2375-2380.

FISCH, N. J. 2010 Transformer recharging with alpha channeling in tokamaks. J. Plasma Phys. 76, $627-634$.

Fisch, N. J. \& Herrmann, M. C. 1994 Utility of extracting alpha particle energy by waves. Nucl. Fusion 34, 1541-1556.

Fisch, N. J. \& Herrmann, M. C. 1995 Alpha power channelling with two waves. Nucl. Fusion 35, $1753-1760$.

FISCH, N. J. \& RAX, J. M. $1992 a$ Current drive by lower hybrid waves in the presence of energetic alpha particles. Nucl. Fusion 32, 549-556.

FISCH, N. J. \& RAX, J. M. $1992 b$ Interaction of energetic alpha particles with intense lower hybrid waves. Phys. Rev. Lett. 69, 612-615.

Herrmann, M. C. \& Fisch, N. J. 1997 Cooling energetic particles in a tokamak with waves. Phys. Rev. Lett. 79, 1495-1498.

Hillairet, J., Voyer, D., Ekedahl, A., Goniche, M., Kazda, M., Meneghini, O., Milanesio, D. \& PREYNAS, M. 2010 ALOHA: an advanced lower hybrid antenna coupling code. Nucl. Fusion 50, 125010.

Hooke, W. 1984 Review of experiments on current drive in tokamaks by means of RF waves. Plasma Phys. Control Fusion 26, 133-144.

Ikeda, Y., Naito, O., Kondoh, T., Seki, M., Ide, S., Asakura, N., Bernabei, S., Fujita, T., ITAMI, K., KAMADA, Y. et al. 1994 High power lower hybrid current drive experiments in JT-60U. In Proceedings of the 15th IAEA Conference on Plasma Physics and Controlled Nuclear Fusion Research, Seville, Spain, vol. 1, p. 415.

Leuterer, F., Söldner, F., Brambilla, M., Munich, M., Monaco, F., Zouhar, M., Bartiromo, R., Tuccillo, A., Bernabei, S. \& Forest, C. 1991 Coupling of the $2 \times 24$ waveguide grill for lower hybrid waves in ASDEX. Plasma Phys. Control. Fusion 33, 169-180. 
LiU, C. S. \& Tripathi, V. K. 1986 Parametric instabilities in a magnetized plasma. Phys. Rep. 130, $143-216$.

LiU, F. K., Ding, B. J., Li, J. G., Wan, B. N., Shan, J. F., Wang, M., LiU, L., Zhao, L. M., LI, M. H., LI, Y. C. et al. 2015 First results of LHCD experiments with $4.6 \mathrm{GHz}$ system toward steady-state plasma in EAST. Nucl. Fusion 55, 123022.

Ochs, I. E., Bertelli, N. \& Fisch, N. J. 2015 a Coupling of alpha channeling to parallel wavenumber upshift in lower hybrid current drive. Phys. Plasmas 22, 082119.

Ochs, I. E., Bertelli, N. \& Fisch, N. J. $2015 b$ Alpha channeling with high-field launch of lower hybrid waves. Phys. Plasmas 22, 112103.

Pavlo, P., Krlin, L. \& Tluchor, Z. 1991 Effects of magnetized alpha particles on lower hybrid heating and current drive in a reactor grade plasma. Nucl. Fusion 31, 711-728.

Pericoli-Ridolfini, V., Ekedahl, A., Erents, S. K., Mailloux, J., Podda, S., Sarazin, Y., TuCCILlo, A. A. \& EFDA-JET WorkPRogramme CONTRIBUtors 2004 Study and optimization of lower hybrid wave coupling in advanced scenario plasmas in JET. Plasma Phys. Control Fusion 46, 349-368.

Pericoli-Ridolfini, V., Giannone, L. \& Bartiromo, R. 1994 Frequency spectral broadening of lower hybrid waves in tokamak plasmas-causes and effects. Nucl. Fusion 34, 469-481.

Petržilka, V. A., Leuterer, F., Söldner, F.-X., Giannone, L. \& Schubert, R. 1991 Nonlinear coupling of the lower hybrid grill in ASDEX. Nucl. Fusion 31, 1758-1767.

Peysson, Y. \& DeCKer, J. 2008 Calculation of $\mathrm{rf}$ current drive in tokamaks. In AIP Conference Proceedings, vol. 1069, pp. 176-187.

Peysson, Y., Decker, J., Morini, L. \& CodA, S. 2011 RF current drive and plasma fluctuations. Plasma Phys. Control Fusion 53, 124028.

Peysson, Y., DeCKer, J.\& Morini, L. 2012 A versatile ray-tracing code for studying rf wave propagation in toroidal magnetized plasmas. Plasma Phys. Control Fusion 54, 045003.

TAKase, Y. \& Porkolab, M. 1983 Parametric excitation of ion-sound quasimodes during lowerhybrid heating experiments in tokamaks. Phys. Fluids 26, 2992-3003.

Takase, Y., Porkolab, M., Schuss, J., Watterson, R., Fiore, C., Slusher, R. \& Surko, C. 1985 Observation of parametric instabilities in the lower-hybrid range of frequencies in the high-density tokamak. Phys. Fluids 28, 983-994.

Tusji, S., Ushigusa, K., Ikeda, Y., Imai, T., Nemoto, M., Nagashima, K., Koide, Y., KaWANO, Y., Fukuda, T., Kondoh, T. et al. 1990 Observation of the limiter $H$ mode in the JT-60 tokamak with lower-hybrid current drive. Phys. Rev. Lett. 64, 1023-1026.

Wallace, G. M., Parker, R. R., Bonoli, P. T., Hubbard, A. E., Hughes, J. W., Labombard, B. L., Meneghini, O., Schmidt, A. E., Shiraiwa, S., Whyte, D. G. et al. 2010 Absorption of lower hybrid waves in the scrape off layer of a diverted tokamak. Phys. Plasmas 17, 082508 .

Wan, B. N., Li, J. G., Guo, H. Y., Liang, Y. F., Xu, G. S., Wang, L., Gong, X. Z., Garofalo, A. \& EAST TEAm AND Collaborators 2015 Advances in H-mode physics for long-pulse operation on EAST. Nucl. Fusion 55, 104015.

WONG, K. L. \& ONO, M. 1984 Effects of ion cyclotron harmonic damping on current drive in the lower hybrid frequency range. Nucl. Fusion 24, 615-626.

Xu, G. S., Wan, B. N., Wang, H. Q., Guo, H. Y., ZhaO, H. L., LiU, A. D., Naulin, V., DiAmond, P. H., TYNAN, G. R., XU, M. et al. 2011 First evidence of the role of zonal flows for the L-H transition at marginal input power in the EAST tokamak. Phys. Rev. Lett. 107, 125001 . 